\title{
The assessment and improvement of the steps in the endoscope-reprocessing: a hospital report
}

\begin{abstract}
Objective: The aim of this study was to describe the endoscope reprocessing procedure of our hospital that succeeded in an effective cleaning that was validated with the microbiological cultures and ATP-bioluminescence tool kit.
\end{abstract}

Material and methods: The endoscope reprocessing procedure was inspected by the microbiological cultures and then ATP-bioluminescence tool kit in the endoscopy units of gastroenterology and general surgery wards of the Ministry of Health Bakırköy Sadi Konuk Research Hospital between April and December 2014.

Results: Of the examined 42 flexible endoscopes, four of them (9\%) were contaminated. In microbiological cultures, Stenotrophomonas maltophilia $(>100,000 \mathrm{cfu} / \mathrm{mL})$ was isolated from irrigation bottles. Extended-spectrum beta-lactamases and plasmid-mediated carbapenamase producing $\mathrm{K}$. pneumonia and $\mathrm{AmpC}$ beta-lactamase producing $P$. aeruginosa $(>100,000 \mathrm{cfu} / \mathrm{mL})$ were isolated from elevator behind. Each step of the reprocessing procedure was inspected and revised, if it was needed. There was not found contamination in the endoscopes after revising procedure.

Conclusion: The ATP-bioluminescence method is a cost-effective method to monitor and to evaluate the endoscope reprocessing. Biofilm formation is very important to harbor resistant microorganisms in the endoscopes. The microbiological monitoring and cleaning procedures of endoscopes should be defined taking into the conditions of the setting in the settings. In case of any outbreak related to endoscopes, each step of the procedure should be checked and revised according to the guidelines, reported outbreaks, and instructions of endoscope manufacturers.

Keywords: endoscopes, ATP, luminescent measurements, disinfection
Volume 5 Issue 3 - 2017

\author{
Habip Gedik \\ Department of Infectious Diseases and Clinical Microbiology, \\ Ministry of Health Bakırköy Sadi Konuk Training and Research \\ Hospital,Turkey
}

\begin{abstract}
Correspondence: Habip Gedik, Associate Professor, Department of Infectious Diseases and Clinical Microbiology, Ministry of Health Bakırköy Sadi Konuk Training and Research Hospital, İstanbul, Turkey, Tel 009050533627 70, Email habipgedik@yahoo.com
\end{abstract}

Received: July 14, 2017 | Published: August 14, 2017

\section{Introduction}

The cleaning and disinfection of flexible endoscopes have been a challenge due to their intricate design, narrow and long lumens, and delicate materials. ${ }^{1}$ They contact with the mucosa and do not penetrate the tissue. They are defined as semi-critical items. ${ }^{2}$ The incidence of healthcare-associated infection related to endoscopes was reported as one per 1.8 million procedures, but the contaminated endoscopes cause outbreaks more than other medical devices. ${ }^{3}$ Endoscopic retrograde cholangiopancreatography (ERCP) duodenoscopes, which are not reprocessed accurately, cause the transmission of microorganisms, including carbapenem-resistant Enterobacteriaceae, Hepatitis B and C virus, HIV, Mycobacterium tuberculosis, and Helicobacter pylori. ${ }^{4,5}$ The validation of cleaning and disinfection by microbiological culture is recommended. ${ }^{5}$ However, the microbiological culture is not easy for all microorganisms, such as viruses and slowly growing pathogens including Helicobacter pylori and $\mathrm{M}$. Tuberculosis, and until endoscope is confirmed to be clean, it cannot be used. Therefore, a more rapid method is needed to inspect the reprocessing of endoscopes. The Gastroenterological Society of Australia and the European Society of Gastroenterology (ESGE)European Society of Gastroenterology and Endoscopy Nurses and Associates (ESGENA) have published guidelines for the surveillance of endoscopes. ${ }^{6,7}$ The FDA recommended supplemental measures to enhance the endoscope-reprocessing with the microbiological culture, and the Centers for Disease Control and Prevention (CDC) published an interim guidelines that recommended the microbiological culture of duodenoscopes in 2015. ${ }^{8,9}$
ATP (adenosine triphosphate) measurement is utilized to monitor cleaning of microbiological contamination. ${ }^{10}$ It measures the quantity of emitted light, when the luciferase enzyme contacts with ATP molecule. ${ }^{11}$ Therefore, ATP measurement is a suitable method to audit rapidly the quality of an endoscope reprocessing. ${ }^{12-15}$

The aim of this study was to describe the endoscope reprocessing procedure of our hospital that succeeded in an effective cleaning that was validated with the microbiological cultures and ATPbioluminescence tool kit.

\section{Materials and methods}

\section{The cleaning and disinfection procedure of hospital}

Each endoscope is disinfected with an enzymatic solution (Cidezyme, Johnson and Johnson Company, İstanbul, Turkey) for one minute followed by a high-level disinfectant $(0.55 \%$ orthophthalaldehyde, Cidex OPA, Johnson and Johnson Company, İstanbul, Turkey) for five minutes after the intervention. At the end of the day, all flexible endoscopes are undergone to manual leakage test and cleaning followed by a mechanical leakage test, cleaning and high-level disinfection in the automated, flexible -endoscope reprocessors (Endoclear, ALX 1011; EndOclear Wiper Device for the Cleaning and Visualization of Endotracheal Tubes, EndOClear LLC, San Ramon, CA) using $0.55 \%$ ortho-phthalaldehyde (OPA). Highlevel disinfectants, such as OPA, inhibit all microorganisms (bacteria, viruses, fungi, mycobacteria), but not bacterial spores. When the disinfection cycle begins, each endoscope is pressurized and tested for 
the leakage. This checks the internal integrity of each endoscope. The pressure is sustained throughout the entire disinfection cycle. In case any defect, such as a hole in the instrument or poor connection seals, is detected, the cycle is automatically stopped. The standard cycle runs for 5minutes and consists of pre-cleaning with an enzymatic cleaner, rinsing, disinfection, rinsing, and air-drying. All cycles are documented on a printed validation label that details the machine serial number, selected cycle, date, time and processed endoscope. These records are attached to the patient list for that day. Details of all endoscopes are also recorded. No research or ethics approval was needed, as this was an in vitro study.

\section{Microbiological sampling and ATP bioluminescence measurement}

Sterile swabs were moisturized with sterile isotonic saline. The biopsy button, elevator behind, a distal end, rinsing valve, endoscope storage cabinets, endoscopy tower were swabbed, and the operating channel, biopsy channel, air-water channel were rinsed with $20 \mathrm{ml}$ sterile isotonic saline. The water samples were taken from the water tank as well. We did not use neutralizers. Swabs and $0.5 \mathrm{ml}$ of rinsing fluid were inoculated onto a 5\% sheep-blood agar (Salubris Inc., Istanbul, Turkey), or a chocolate agar (Salubris Inc., Istanbul, Turkey) and a MacConkey agar (Salubris Inc., Istanbul, Turkey), respectively. Bacterial species were identified by the Phoenix automated microbiology system (BD Diagnostic Systems, Sparks, $\mathrm{MD}$ ), followed by routine microbial laboratory proceedings. Bacterial growth was defined as the number of colony forming unit (CFU). The ATP-bioluminescence was determined using $3 \mathrm{M}^{\mathrm{TM}}$ Clean-Trace ${ }^{\mathrm{TM}}$ Hygiene Monitoring System (MN, USA). The measurement was carried out according to the instructions of manufacturer immediately after swabbing. Reagent blanks were obtained using sterile swabs moisturized with sterile isotonic saline instrument disinfectant and endoscope cleaner used for endoscope reprocessing. Bioluminescence readings were expressed as the relative light units (RLU). Lower than 200 RLU was defined as the clean according to manufacturer's instructions. ${ }^{12,16}$

\section{Results}

Between April and December 2014, we examined 42 flexible endoscopes (made by Pentax, Olympus, Fujinon and Storz) in the gastroenterology and general surgery endoscopy units after reprocessing. In both units, approximately 60 endoscopic interventions were performed in both units in a day. There were 10 automated endoscope-reprocessors in both units. There were five water tanks, two endoscopy towers, and eight endoscope-storage cabinets. The number of contaminated endoscopes was four $(9 \%)$. In the microbiological evaluation, Stenotrophomonas maltophilia $(>100,000 \mathrm{cfu} / \mathrm{mL})$ was isolated from the irrigation bottles. Extendedspectrum beta-lactamases and plasmid-mediated carbapenamase producing $\mathrm{K}$. pneumonia and $\mathrm{AmpC}$ beta-lactamase producing $P$. aeruginosa $(>100,000 \mathrm{cfu} / \mathrm{mL})$ were isolated from the elevator behind. AmpC beta-lactamase producing P. aeruginosa $(>100,000$ $\mathrm{cfu} / \mathrm{mL}$ ) was isolated from the outer surface of a gastroscopy. Diphtheroid bacilli and methicillin-sensitive Staphylococcus aureus were isolated from the endoscope hanger. After the extension of disinfection time to 10minutes and re-disinfection of all equipments before the intervention in the morning, control cultures did not yield any microorganism. However, 42 endoscopes and approximately 400 microbiological cultures caused a remarkable workload and costs. The infection control committee decided to use the ATP-bioluminescence tool for the microbiological monitoring of endoscopes. It results in 30seconds, and prevents out of use of the endoscopes until cultures are concluded. The endoscope-reprocessing procedure was revised according to the guidelines and reported studies:

I. The cleaning and reprocessing of the endoscopes will be inspected and be monitored by ATP-bioluminescence tool every threemonths.

II. Disinfection is required when the waiting time of endoscopes exceeds 12 hours after washing.

III. The bottles and connecting hoses used for endoscope irrigation will be cleaned and sterilized. Sterile water is used during the procedure.

IV. The elevator wire channel of endoscopes could need to be washed using the syringe, as the flushing pressure could be insufficient.

$\mathrm{V}$. The non-foaming detergents at the neutral $\mathrm{pH}$ or the enzymatic cleaners are recommended by guidelines. Enzymatic cleaners are recommended instead of $\mathrm{pH}$-unknown detergents.

VI. The manufacturer should instruct the effective time of enzymatic cleaners for the devices.

VII. The contact time of $0.55 \%$ ortho-phthalaldehyde (OPA) has been increased to 10 minutes.

VIII. The endoscopes are placed on a flat surface and aired through the air duct with an injector after completion of disinfection. High pressure, which could damage the gun channel was used only at the end portions. In addition, elevator inside and back should be dried carefully with a sponge.

IX. The elevator in the duodenoscopy channel can hamper the brushing. The washing, enzymatic cleaning, drying should be performed with a syringe. After placement of the disinfected connection hose into the elevator channel with a suitable adapter, the disinfection, rinsing, and drying should be performed. The diameter of the channel is too small, so maximum $5 \mathrm{cc}$ syringe was used. ${ }^{-6,611}$

After implementation of those steps, we achieved the accurate endoscope-reprocessing validated by the ATP-bioluminescence tool and no infection-related complication developed.

\section{Discussion}

Endoscopes are the temperature sensitive items, so lowtemperature chemical methods, such as liquid chemical germicide, must be used rather than steam sterilization. In the guidelines, the manual washing, high level disinfection with the automated endoscope reprocessing and drying was emphasized for the infection prevention and control regarding gastrointestinal (GI) system endoscopy. ${ }^{17}$. Inadequate cleaning and disinfection are more linked to GI-endoscopes related outbreaks compare to other medical devices. The number of contaminated endoscopes in our investigation (9\%) is less than those reported on the results of Moses and Lee that from $12 \%$ to $24 \%$ of the microbiological cultures yielded microorganisms during a 10 -year study period..$^{15}$ Moses and Lee examined only endoscopes used in a clinical institution and reprocessed in an automated washer. They used the microbiological culture method for the surveillance, whereas culture and ATP-bioluminescence methods were used in our investigation. The ATP-bioluminescence tool provides the fast and cost-effective microbiological monitoring of 
endoscopes in endoscopy units where the number of daily endoscopic implementation is high. The monitoring of endoscope reprocessing to prevent and control of healthcare associated infections is an essential component of safe endoscopy applications. Because the endoscope reprocessing is a multistep procedure affecting its efficacy. However, there is no consensus on the frequency of routine microbiological testing of endoscopes. ${ }^{17,18}$ The frequency of microbiological testing of the endoscopes was reported to be once every 3 to 6 months according to guidelines. ${ }^{17}$ The microbial growth was reported to be in $5.0 \%$ of the specimens that yielded the environmental microbes. Enteric bacteria were isolated in 6\% of the specimens, although the 2014 guidelines of the USA and the instructions of manufacturers regarding the cleaning and high-level disinfection process were fulfilled accurately. ${ }^{18}$ Therefore, the microbiological monitoring procedure should be defined in each setting inspecting each step of cleaning and disinfection.

There are several disinfectants with their advantages and disadvantages. Short contact time is a major pitfall because of the intensive use of endoscopes. Glutaraldehyde solution (2\%) is the most commonly used disinfectant in GI endoscope reprocessing with an at least 20minutes of contact time, whereas the OPA is a high-level disinfectant, and more expensive solution than the glutaraldehyde solution. It has a contact time that varies from 5 to 12 minutes in different countries. The contact time of the OPA $(0.55 \%)$ is between 5-12minutes in Europe, Latin America, and Asia; 5minutes in Canada; 10minutes in Australia; and 12minutes in the USA as a. ${ }^{19,20}$ When the contact time of the OPA was extended to 10 minutes, the disinfection of endoscopes became accurate in our procedure.

The bottles for the irrigation and connecting the hoses might be contaminated and colonized during the implementation. They might harbor the resistant microorganisms, such as $P$. aeruginosa, Acinetobacter spp. surviving for a long time in the humid environment, if they are not sterilized accurately. Biofilm formation constitutes especially a nidus for the resistant microorganisms in the connection hoses. Mechanical cleaning is an important step to remove the nidus. ${ }^{1-4,20}$ It was reported that neither the detergent nor the high-level disinfectants could remove or kill the bacteria at the expected level due to the biofilm formation in the channels. ${ }^{21,22}$ Detergents and enzymatic cleaners cannot remove the biofilm in the endoscopes sufficiently, so the mechanical cleaning must be definitely performed. The syringe should be used, as the flushing pressure might be insufficient and the debris might not be removed exactly. The preparation of enzymatic cleaner for the cleaning of the endoscopes by staff should be inspected and monitored. The effective time and usage of enzymatic cleaners should be defined and instructed to authorized staff according to the instructions of the manufacturers regarding the cleaners and devices. The non-foaming detergents at the neutral $\mathrm{pH}$ or the enzymatic cleaners prevent corrosion in the endoscopes that might be nidus for microorganisms and their biofilm formations. Air ducts should be aired within an appropriate pressure to dry and to prevent the biofilm formation under humid conditions. The elevating mechanism causes the failure of reprocessing. Olympus has recently designed a smaller brush to improve the elevator cleaning addressing that problem. Flocked swabs were reported to capture and to release organisms more efficiently than the cleaning brushes. ${ }^{23}$

This study has several potential limitations. First, we used a qualitative method to evaluate the uncleanliness of the endoscopes. Two methods were not compared in terms of their effectiveness, but they were used for the assessment. We have inspected the each step in the disinfection and cleaning of the endoscopes to find failures in this study without emphasizing any method and guideline. However, no standard method has been established for the assessment of the endoscope reprocessing. ${ }^{24}$

As a result, the ATP-bioluminescence tool is a cost-effective method to inspect and to monitor the endoscope reprocessing. Biofilm formation is very important to harbor resistant microorganisms in the endoscopes. The microbiological monitoring and cleaning procedure of the endoscopes should be defined at each setting with its frequency. In case of any outbreak related to endoscopes, each step of the reprocessing should be inspected and be corrected taking into guidelines, reported outbreaks, as well as instructions of endoscope manufacturers.

\section{Acknowledgements}

None.

\section{Conflict of interest}

The author declares no conflict of interest.

\section{References}

1. Martiny H, Floss H, Zuhlsdorf B. The importance of cleaning for the overall results of processing endoscopes. J Hosp Infect. 2004;56(Suppl 2):S16-S22.

2. Leung J, Vallero R, Wilson R. Surveillance cultures to monitor quality of gastrointestinal endoscope reprocessing. Am $J$ Gastroenterol. 2003;9(1):3-5.

3. Leung JW. Reprocessing of flexible endoscopes. $J$ Gastroenterol Hepatol. 2000;15(Suppl):G73-G77.

4. Epstein L, Hunter JC, Arwady MA, et al. New Delhi metallo-betalactamase-producing carbapenem-resistant Escherichia coli associated with exposure to duodenoscopes. JAMA. 2014;312(14):1447-1455.

5. Verantwortlicher Redakteur, Sophia Seidel. Bundesgesundheitsblatt Gesundheitsforschung Gesundheitsschutz. Family \& Geriatric Medicine. 2002;45(4):395-411.

6. Beilenhoff U, Neumann CS, Rey JF, et al. ESGE-ESGENA guideline for quality assurance in reprocessing:microbiological surveillance testing in endoscopy. Endoscopy. 2007;39:175-181.

7. Whitby M, Fielding D, Todman M, et al. Infection control in endoscopy. 3rd ed. Australia: Gastroenterological Society of Australia; 2010.

8. United States Food and Drug Administration. FDA safety communication: supplemental measures to enhance duodenoscope reprocessing. 2015.

9. Centers for Disease Control and Prevention. Interim sampling method for the duodenoscope: distal end and instrument channel. GA: Centers for Disease Control and Prevention; 2015.

10. Seeger K, Griffiths MW. Adenosine triphosphate bioluminescence for hygiene monitoring in health care institutions. $J$ Food Prot. 1994;57(6):509-512.

11. Baumgart J. Möglichkeiten und Grenzen moderner Schnellverfahren zur Prozesskontrolle von Reinigungs- und Desinfektionsverfahren. Zentralbl Hyg Umweltmed. 1996;199(2-4):366-375.

12. Alfa MJ, Fatima I, Olson N. Validation of adenosine triphosphate to audit manual cleaning of flexible endoscope channels. Am J Infect Control. 2013;41(3):245-248

13. Fushimi R, Takashina M, Yoshikawa H, et al. Comparison of adenosine triphosphate, microbiological load, and residual protein as indicators for assessing the cleanliness of flexible gastrointestinal endoscopes. Am J Infect Control. 2012;41(2):161-164. 
14. Shin SP, Kim WH. Recent Update on Microbiological Monitoring of Gastrointestinal Endoscopes after High-Level Disinfection. Clin Endosc. 2015;48(5):369-373.

15. Moses FM, Lee J. Surveillance cultures to monitor quality of gastrointestinal endoscope reprocessing. $\mathrm{Am} J$ Gastroenterol. 2003;98(1):77-81.

16. Alfa MJ, Fatima I, Olson N. The adenosine triphosphate test is a rapid and reliable audit tool to assess manual cleaning adequacy of flexible endoscope channels. Am J Infect Control. 2013;41(3):249-253.

17. Gillespie EE, Kotsanas D, Stuart RL. Microbiological monitoring of endoscopes:5-year review. J Gastroenterol Hepatol. 2008;23(7 pt 1):1069-1074

18. Brandabur JJ, Leggett JE, Wang L, et al. Surveillance of guideline practices for duodenoscope and linear echoendoscope reprocessing in a largehealthcare system. Gastrointest Endosc. 2016;84(3):392-399.

19. Petersen BT, Chennat J, Cohen J, et al. Multisociety guideline on reprocessing flexible GI endoscopes: 2011. Infect Control Hosp Epidemiol. 2011;32:527-537.
20. Augustin M, Ali-Vehmas T, Atroshi F. Assessment of enzymatic cleaning agents and disinfectants against bacterial biofilms. J Pharm Pharm Sci. 2004;7(1):55-64.

21. da Costa Luciano C, Olson N, Tipple AF, et al. Evaluation of the ability of different detergents and disinfectants to remove and kill organisms in traditional biofilm. Am J Infect Control. 2016;44(11):e243-e249.

22. da Costa Luciano C, Olson N, DeGagne P, et al. A new buildup biofilm model that mimics accumulation of material in flexibe endoscope channels. J Microbiol Methods. 2016;127:224-229.

23. Gazdik MA, Coombs J, Burke JP, et al. Comparison of Two Culture Methods for use in Assessing Microbial Contamination of Duodenoscopes. J Clin Microbiol. 2016;54(2):312-316.

24. Rutala WA, Weber DJ. Healthcare Infection Control Practices Advisory Committee (HICPAC). Guideline for Disinfection and Sterilization in Healthcare Facilities. 2008. 